\title{
Scale invariant events and dry spells for medium-resolution local rain data
}

\author{
A. Deluca ${ }^{1,2}$ and Á. Corral ${ }^{1,2}$ \\ ${ }^{1}$ Centre de Recerca Matemàtica, Edifici Cc, Campus Bellaterra, 08193 Bellaterra, Barcelona, Spain \\ ${ }^{2}$ Departament de Matemàtiques, Universitat Autònoma de Barcelona, 08193 Bellaterra, Barcelona, Spain
}

Correspondence to: A. Deluca (adeluca@crm.cat) and Á. Corral (acorral@crm.cat)

Received: 12 May 2012 - Revised: 7 February 2014 - Accepted: 14 February 2014 - Published: 25 April 2014

\begin{abstract}
We analyze distributions of rain-event sizes, rainevent durations, and dry-spell durations for data obtained from a network of 20 rain gauges scattered in a region of the northwestern Mediterranean coast. While power-law distributions model the dry-spell durations with a common exponent $1.50 \pm 0.05$, density analysis is inconclusive for event sizes and event durations, due to finite size effects. However, we present alternative evidence of the existence of scale invariance in these distributions by means of different data collapses of the distributions. These results demonstrate that scaling properties of rain events and dry spells can also be observed for medium-resolution rain data.
\end{abstract}

\section{Introduction}

The complex atmospheric processes related to precipitation and convection arise from the cooperation of diverse non-linear mechanisms with different temporal and spatial characteristic scales. Precipitation combines, for instance, the $\mathcal{O}(100 \mu \mathrm{m})$ microphysics effects as evaporation with $\mathcal{O}(1000 \mathrm{~km})$ planetary circulation of masses and moisture (Bodenschatz et al., 2010). Rain fields also present high spatial and temporal intermittency as well as extreme variability, in such a way that their intensity cannot be characterized by its mean value. Despite the complexity of the processes involved, surprising statistical regularities have been found: numerous geometric and radiative properties of clouds present clear scaling or multiscaling behavior (Lovejoy, 1982; Cahalan and Joseph, 1989; Peters et al., 2009; Wood and Field, 2011); also, raindrop arrival times and raindrop sizes, are well characterized by power-law distributions over several of orders of magnitude (Olsson et al., 1993; Lavergnat and Golé, 2006).
The concept of self-organized criticality (SOC) aims for explaining the origin of the emergence of structures across many different spatial and temporal scales in a broad variety of systems (Bak, 1996; Jensen, 1998; Sornette, 2004; Christensen and Moloney, 2005). Indeed, it has been found that for diverse phenomena that take place intermittently, in terms of bursts of activity interrupting larger quiet periods, the size, $s$, of these bursty events or avalanches follows a power-law distribution,

$$
P_{S}(s) \propto \frac{1}{s^{\tau_{s}}},
$$

over a certain range of $s$, where $P_{S}(s)$ is the probability density of the event size and $\tau_{s}$ its exponent (and the sign $\propto$ indicating proportionality). The size, $s$, can be understood as a measure of energy dissipation. If durations of events are measured, a power-law distribution also holds. These power-law distributions provide an unambiguous proof of the absence of characteristic scales within the avalanches, as power laws are the signature of scale invariance (Christensen and Moloney, 2005).

The main idea behind SOC is the claim that such scale invariance is achieved because of the existence of a nonequilibrium continuous phase transition whose critical point is an attractor of the dynamics when the system is slowly driven (Tang and Bak, 1988; Dickman et al., 1998, 2000). When the system settles at the critical point, scale invariance and power-law behavior are ensured, as these peculiarities are the defining characteristics of critical phenomena (Christensen and Moloney, 2005). SOC has had a big impact in the geosciences, in particular earthquakes (Bak, 1996; Sornette and Sornette, 1989), landslides and rock avalanches (Malamud, 2004), or forest fires (Malamud et al., 1998). Due to the existence of power-law distributed events in them, 
these systems have been proposed as realizations of SOC in the natural world; nevertheless, no evidence of an underlying critical point has been presented. On the other hand, it is also possible to understand SOC in a broader sense, as the spontaneous emergence of scale invariance; therefore, SOC is not considered a mechanism based on an attraction towards a continuous phase transition, but arising from a different phenomenology, where stochasticity and non-vanishing fluxes play a key role, as it happens in multifractal cascades (Schertzer and Lovejoy, 1994, 2011; Hooge et al., 1994; Lovejoy and Schertzer, 2013).

SOC observables have been explored in rainfall while looking at precipitation as an avalanche process, and paying attention to the properties of these avalanches, called rain events. To our knowledge, the first works following this approach are those of Andrade et al. (1998) and Peters et al. (2002) (Peters and Christensen, 2002, 2006). These authors defined, independently, a rain event as the sequence of rain occurrence with a rain rate (i.e., the activity) always greater than zero. Therefore, the focus of these approaches is not on the total amount of rain recorded in a fixed time period (for instance, one hour, one day, or one month), but on the rain event, which is what defines in each case the time period of rain-amount integration. In this way, the event size is the total amount of rain collected during the duration of the event.

Andrade et al. (1998) studied long-term daily local (i.e., zero dimensional) rain records from weather stations in Brazil, India, Europe, and Australia, with observation times ranging from a decade to a century approximately, with detection threshold $0.1 \mathrm{mmday}^{-1}$. Although the dry spells (the times between rain events) seemed to follow in some case a steep power-law distribution, the rain-event size distributions were not reported, and therefore the connection between SOC and rainfall could not really be checked. Later, Peters et al. (2002) analyzed high resolution rain data from a vertically pointing Doppler radar situated on the Baltic coast, which provided rates at an altitude between 250 and 300 ma.s.l., covering an area of $70 \mathrm{~m}^{2}$, with detection threshold $0.0005 \mathrm{~mm} \mathrm{~h}^{-1}$ and temporal resolution of $1 \mathrm{~min}$. Powerlaw distributions for event sizes and for dry-spell durations over several orders of magnitude were reported, with exponents $\tau_{s} \simeq \tau_{q} \simeq 1$.4. For the event-duration distribution the results were unclear, although a power law with an exponent $\tau_{d} \simeq 1.6$ was fit to the data. More recently, a study covering 10 sites across different climates has checked the universality of rain-event statistics using rain data from optical gauges (Peters et al., 2010). The data had a detection threshold of $0.2 \mathrm{mmh}^{-1}$, and were collected at intervals of $1 \mathrm{~min}$. The results showed unambiguous power-law distributions of event sizes, with apparent universal exponents $\tau_{s}=1.17 \pm 0.03$. Power-law distributions were also found for the dry spell durations, but for event durations the behavior was not conclusive.

Nevertheless, scale-free distributions of the avalanche-like observables are insufficient evidence for "classical" SOC dy- namics, as there are many alternative mechanisms of powerlaw genesis (Sornette, 2004; Dickman, 2003). In other words, SOC implies power laws, but the reciprocal is not true, power laws are not a guarantee of SOC. Of particular relevance is the multifractal approach, which can also reproduce scale invariance but using different observables. When applied to rainfall, this approach focus on the rain rate field, which is hypothesized to have multifractal support as a result from a multiplicative cascade process. From this point of view, alternative statistical models and new forecasting and downscaling methods have emerged (Schertzer and Lovejoy, 1992; Lovejoy and Schertzer, 1995; Deidda, 2000; Veneziano and Lepore, 2012).

We will study the scaling of the distributions of event observables without ascribing this scaling to SOC or any other mechanism. As we have seen, the number of studies addressing this kind of scaling is rather limited, mostly due to the supposed requirement that the data has to be of very high time and rate resolution. The goal of the paper is to extend the evidence for power laws in rainfall, in particular in the distribution of the observables related to rain events, studying the applicability of this paradigm when the rain data available is not of high resolution. With this purpose, we perform an in-depth analysis of local rainfall records in a representative region of the northwestern Mediterranean. For this lower (in comparison with previous studies) resolution, the range in which the power law holds can be substantially decreased. This may require the application of more refined fitting techniques and scaling methods. Thus, as a byproduct, we explore different scaling forms and develop a collapse method based on minimizing the distance between distributions that also gives an estimation of the power-law exponent. With these tools we will be able to establish the existence of scale-invariant behavior in the medium-resolution rain data analyzed.

We proceed as follows: Sect. 2 describes the data used in the present analysis and defines the rain event, its size and duration, and the dry spell. Section 3 shows the corresponding probability densities and describes and applies an accurate fitting technique for evaluating the power-law existence. Section 4 introduces two collapse methods (parametric and non-parametric) in order to establish the fulfillment of scaling, independently of power-law fitting. Discussion and conclusions are presented in Sect. 5.

\section{Data and definitions}

\subsection{Data}

We have analyzed 20 stations in Catalonia (NE Spain) from the database maintained by the Agència Catalana de l'Aigua (ACA, 2014). These data come from a network of rain gauges, called SICAT (Sistema Integral del Cicle de l'Aigua al Territori, formerly SAIH, Sistema Automàtic d'Informació 
Table 1. Characteristics of all the sites for the 9-year period 2000-2008. Every site is named by the corresponding river basin or subbasin (the municipality is included in ambiguous cases); Ll. stands for Llobregat river; $f_{\mathrm{M}}$ is the fraction (in \%) of missing records (time missing divided by total time); $f_{\mathrm{D}}$ is the fraction (in $\%$ ) of discarded times; $f_{r}$ is the fraction (in $\%$ ) of rainy time (time with $r>0$ divided by total non-discarded time, for a time resolution $\Delta t=5 \mathrm{~min}$ ); a. rate is the annual rain rate in mm year ${ }^{-1}$, calculated only over non-discarded times; c. rate is the rain rate in $\mathrm{mm} \mathrm{h}^{-1}$ conditioned to rain, i.e., calculated over the (non-discarded) rainy time; $N_{S}$ is the number of rain events and $N_{q}$ the number of dry spells (the differences between $N_{s}$ and $N_{q}$ are due to the missing records); the rest of symbols are explained in the text. $\langle s\rangle$ is measured in $\mathrm{mm}$, and $\langle d\rangle$ and $\langle q\rangle$ in min. Sites are ordered by increasing annual rate. The table shows a positive correlation between $f_{r}$, the annual rate, $N_{s}$ and $N_{q}$, and that these variables are negatively correlated with $\langle q\rangle$. In contrast, the rate conditioned to rain is roughly constant, taking values between 3.3 and $3.8 \mathrm{~mm} \mathrm{~h}^{-1}$.

\begin{tabular}{lcrcccccccc}
\hline Site & $f_{\mathrm{M}}$ & $f_{\mathrm{D}}$ & $f_{r}$ & a.rate & c. rate & $N_{s}$ & $N_{q}$ & $\langle s\rangle$ & $\langle d\rangle$ & $\langle q\rangle$ \\
\hline 1 Gaià & 0.08 & 3.71 & 1.6 & 470.9 & 3.3 & 5021 & 5014 & 0.81 & 14.9 & 894 \\
2 Foix & 0.07 & 3.38 & 1.6 & 500.6 & 3.6 & 4850 & 4844 & 0.90 & 15.0 & 929 \\
3 Baix Ll. S.J. Despí & 0.07 & 2.28 & 1.7 & 505.8 & 3.3 & 5374 & 5369 & 0.83 & 15.0 & 847 \\
4 Garraf & 0.09 & 3.30 & 1.6 & 507.8 & 3.7 & 4722 & 4716 & 0.94 & 15.2 & 956 \\
5 Baix Ll. Castellbell & 0.06 & 2.81 & 1.7 & 510.7 & 3.4 & 4950 & 4947 & 0.90 & 15.8 & 914 \\
6 Francolí & 0.44 & 13.37 & 1.8 & 528.2 & 3.4 & 4539 & 4540 & 0.91 & 16.1 & 887 \\
7 Besòs Barcelona & 0.15 & 4.17 & 1.7 & 531.8 & 3.5 & 4808 & 4803 & 0.95 & 16.2 & 928 \\
8 Riera de La Bisbal & 0.07 & 3.66 & 1.6 & 540.0 & 3.8 & 4730 & 4724 & 0.99 & 15.8 & 950 \\
9 Besòs Castellar & 4.34 & 13.59 & 2.0 & 633.3 & 3.6 & 4918 & 4970 & 1.00 & 16.9 & 806 \\
10 Ll. Cardener & 0.07 & 3.33 & 2.1 & 652.4 & 3.5 & 6204 & 6197 & 0.92 & 15.7 & 723 \\
11 Ridaura & 0.12 & 2.41 & 2.0 & 674.2 & 3.8 & 5780 & 5774 & 1.02 & 16.1 & 784 \\
12 Daró & 0.06 & 2.09 & 2.2 & 684.5 & 3.6 & 5553 & 5547 & 1.09 & 18.0 & 818 \\
13 Tordera & 0.08 & 2.04 & 2.3 & 688.8 & 3.4 & 7980 & 7977 & 0.76 & 13.6 & 568 \\
14 Baix Ter & 0.07 & 2.71 & 2.3 & 710.2 & 3.6 & 6042 & 6036 & 1.03 & 17.4 & 746 \\
15 Cap de Creus & 0.07 & 2.92 & 2.3 & 741.5 & 3.7 & 5962 & 5955 & 1.09 & 17.7 & 754 \\
16 Alt Llobregat & 3.12 & 5.82 & 2.6 & 742.8 & 3.3 & 6970 & 6988 & 0.90 & 16.7 & 621 \\
17 Muga & 0.06 & 2.56 & 2.4 & 749.3 & 3.6 & 6462 & 6457 & 1.02 & 16.9 & 698 \\
18 Alt Ter Sau & 0.08 & 2.43 & 2.5 & 772.1 & 3.6 & 6966 & 6961 & 0.97 & 16.3 & 647 \\
19 Fluvià & 3.09 & 4.74 & 2.3 & 772.4 & 3.8 & 6287 & 6319 & 1.05 & 16.7 & 697 \\
20 Alt Ter S. Joan & 0.07 & 1.98 & 2.8 & 795.1 & 3.3 & 8333 & 8327 & 0.84 & 15.5 & 452 \\
\hline
\end{tabular}

Hidrològica), used to monitor the state of the drainage basins of the rivers that are born and die in the Catalan territory. The corresponding sites are listed in Table 1 and have longitudes and latitudes ranging from $1^{\circ} 10^{\prime} 51^{\prime \prime}$ to $3^{\circ} 7^{\prime} 35^{\prime \prime} \mathrm{E}$ and from $41^{\circ} 12^{\prime} 53^{\prime \prime}$ to $43^{\circ} 25^{\prime} 40^{\prime \prime} \mathrm{N}$. All data sets cover a time period starting on 1 January 2000, at 00:00 LT, and ending either on 30 June or on 1 July 2009 (spanning roughly 9.5 years), except the Cap de Creus, which ends on 19 June 2009.

In all the stations, rain is measured by the same weighing precipitation gauge, the device called Pluvio from OTT Hydromet $\mathrm{GmbH}$, either with a capacity of 250 or $1000 \mathrm{~mm}$ and working through the balance principle. It measures both liquid or/and solid precipitation. The precipitation rate is recorded in intervals of $\Delta t=5 \mathrm{~min}$, with a resolution of $1.2 \mathrm{mmh}^{-1}$ (which corresponds to $0.1 \mathrm{~mm}$ in $5 \mathrm{~min}$ ). This precipitation rate can be converted into an energy flux through the latent heat of condensation of water, which yields $1 \mathrm{mmh}^{-1} \simeq 690 \mathrm{Wm}^{-2}$, nevertheless, we have not performed such conversion. Figure 1a shows a subset of the time series for site 17 (Muga).
In order to make the files more manageable, the database reports zero-rain rates only every hour; then we consider time voids larger than $1 \mathrm{~h}$ as operational errors. The ratio of these missing times to the total time covered in the record is denoted as $f_{\mathrm{M}}$ in Table 1, where it can be seen that this is usually below $0.1 \%$. However, there are three cases in which its value is around 3 or $4 \%$. Other quantities reported in the table are the fraction of time corresponding to rain (or wet fraction), $f_{r}$, the annual mean rate, and the mean rate conditioned to rain periods. Nevertheless, note that for a fractal point process a quantity as $f_{r}$ depends on the time resolution; so, $f_{r}$ only makes sense for a concrete time division, in our case, $\Delta t=5 \mathrm{~min}$.

\subsection{Rain event sizes, rain event durations, and dry spell durations}

Following Andrade et al. (1998) and Peters et al. (2002), we define a rain event as a sequence of consecutive rain rates bigger than zero delimited by zero rates, i.e., $\left\{r\left(t_{n}\right), r\left(t_{n+1}\right), \ldots r\left(t_{m}\right)\right\}$, such that $r\left(t_{i}\right)>0$ for $i=n, n+$ $1, \ldots m$ with $r\left(t_{n-1}\right)=r\left(t_{m+1}\right)=0$. Due to the resolution of 
the record, this is equivalent to take a threshold with a value below $1.2 \mathrm{mmh}^{-1}$. It is worth mentioning that this simple definition of rain events may be in conflict with those used by the hydrologist community, so caution is required in order to make comparisons between the different approaches (Molini et al., 2011).

The first observable to consider is the duration, $d$, of the event, which is the time that the event lasts (a multiple of $5 \mathrm{~min}$, in our case). The size of the event is defined as the total rain during the event (i.e., the rate integrated over the event duration),

$s \equiv \sum_{i=n}^{m} r\left(t_{i}\right) \Delta t \simeq \int_{t_{n}}^{t_{m}} r(t) d t$

measured in $\mathrm{mm}$ (and multiple of $0.1 \mathrm{~mm}$ in our case, $\left.1.2 \mathrm{mmh}^{-1} \times 5 \mathrm{~min}\right)$. Notice that this event size is not the same as the usual rain depth, due to the different definition of the rain event in each case. Figure 1b shows as an illustration the evolution of the rate for the largest event in the record, which happens at the Muga site, whereas Fig. 1c displays the sequence of all event sizes in the same site for the year 2002. It is important to realize that this quantity is different to the one at Fig. 1a. Regarding event durations, the time series have a certain resemblance to Fig. 1c, as usually they are (nonlinearly) correlated with event sizes (Telesca et al., 2007). Further, the dry spells are the periods between consecutive rain events (then, they verify $r(t)=0$ ); we denote their durations by $q$. When a rain event, or a dry spell, is interrupted due to missing data, we discard that event or dry spell, and count the recorded duration as discarded time; the fraction of these times in the record appears in Table 1, under the symbol $f_{\mathrm{D}}$. Although in some cases the duration of the interrupted event or dry spell can be bounded from below or from above (as in censored data), we have not attempted to use that partial information.

\section{Power-law distributions}

\subsection{Probability densities}

Due to the enormous variability of the three quantities just defined, the most informative approach is to work with their probability distributions. Taking the size as an example, its probability density, $P_{S}(s)$, is defined as the probability that the size is between $s$ and $s+d s$ divided by $d s$, with $d s \rightarrow 0$. Subsequently, $\int_{0}^{\infty} P_{S}(s) d s=1$. This implicitly assumes that $s$ is considered as a continuous variable (but this will be corrected later, see more details on Appendix A). In general, we illustrate all quantities with the event size $s$, the analogous for $d$ and $q$ are obtained by replacing $s$ with the symbol of each observable. The corresponding probability densities are denoted as $P_{\mathrm{D}}(d)$ and $P_{Q}(q)$. Note that the annual number densities (Peters et al., 2002; Peters and Christensen, 2002,
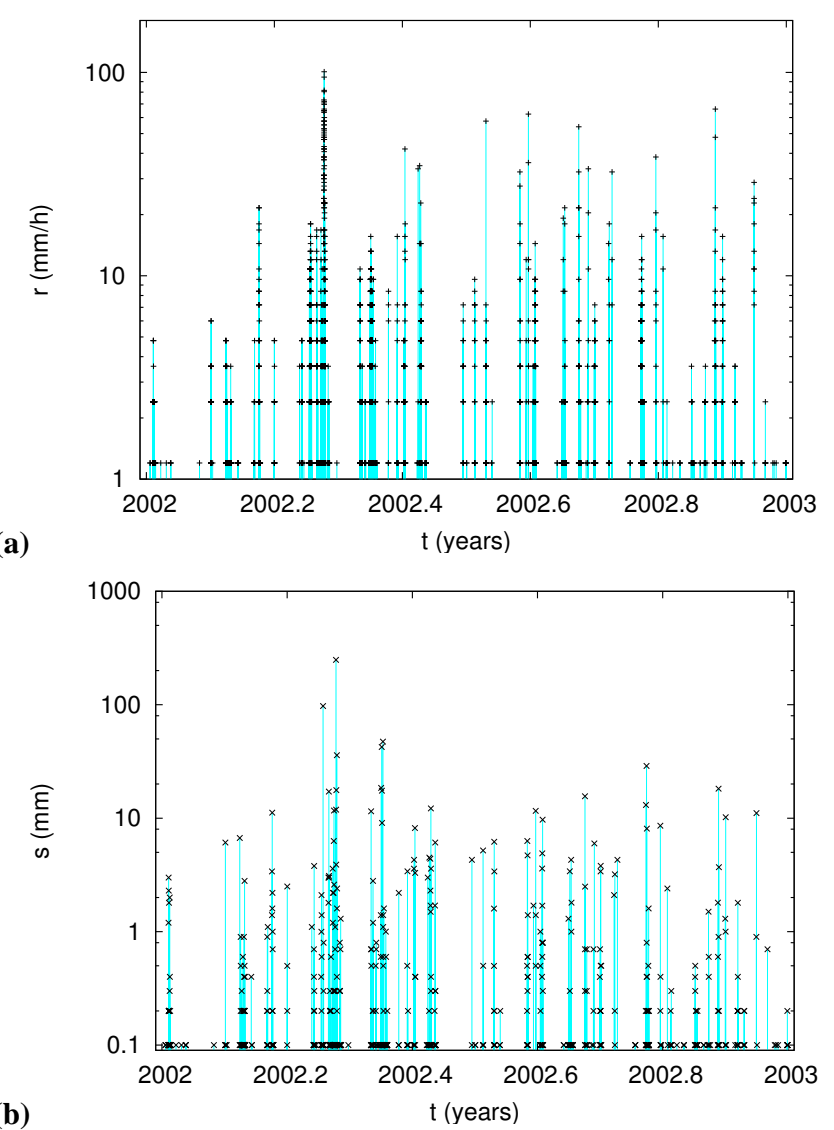

(b)

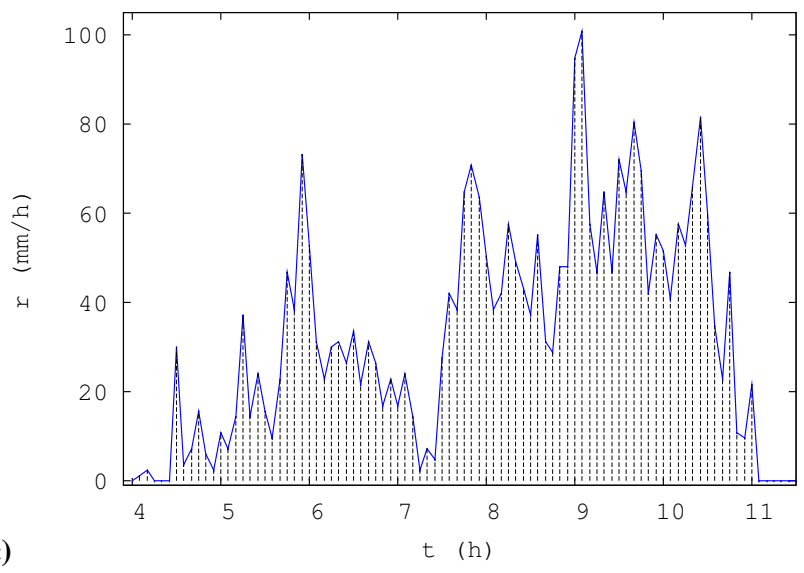

Fig. 1. (a) Subset of the rain rate time series for site 17 (Muga) for year 2002. (b) More reduced subset of the rain rate time series for the same site, corresponding to the largest rain event on the record, with $s=248.7 \mathrm{~mm}$, on 11 April 2002. Time refers to hours since midnight. A very small rain event is also present at the beginning, with $s=0.3 \mathrm{~mm}$ and separated to the main event by a dry spell of duration $q=15 \mathrm{~min}$. (c) Corresponding event-size time series for the same site, for year 2002 . 
2006) are trivially recovered multiplying the probability densities by the total number of events or dry spells and dividing by total time.

The results for the probability densities $P_{S}(s), P_{\mathrm{D}}(d)$, and $P_{Q}(q)$ of all the sites under study are shown in Fig. 2a, $\mathrm{b}$, and c, respectively. In all cases the distributions show a very clear behavior, monotonically decreasing and covering a broad range of values. However, to the naked eye, a powerlaw range is only apparent for the distributions of dry spells, $P_{Q}(q)$ (remember that a power law turns into a straight line in a log-log plot). Moreover, the $P_{Q}(q)$ are the broadest distributions, covering a range of more than 4 orders of magnitude (from $5 \mathrm{~min}$ to about a couple of months), and present in some cases a modest daily peak (in comparison to Peters et al., 2002, with 1 day $=1440 \mathrm{~min}$ ). In the opposite side, we find the distributions of durations, $P_{\mathrm{D}}(d)$, whose range is the shortest, from $5 \mathrm{~min}$ to about 1 day (two and a half orders of magnitude), and for which no straight line is visible in the plot; rather, the distributions appear as convex. The size distributions, $P_{S}(s)$, defined for about 3 orders of magnitude (from 0.1 to $200 \mathrm{~mm}$ roughly), can be considered in between the other two cases, with a visually shorter range of powerlaw behavior.

\subsection{Fitting and testing power laws}

A quantitative method can put more rigor into these observations. The idea is based on the recipe proposed by Clauset et al. (2009) - see also Corral et al. (2011) - but improved and generalized to our problem. Essentially, an objective procedure is required in order to find the optimum range in which a power law may hold. Taking again the event size for illustration, we report the power-law exponent fit between the values of $s_{\min }$ and $s_{\max }$ which yield the maximum number of data in that range but with a $p$ value greater than $10 \%$. The method is described in Peters et al. (2010) and in more detail in Deluca and Corral (2013), but we summarize it in the following paragraphs.

For a given value of the pair $s_{\min }$ and $s_{\max }$, the maximumlikelihood (ML) power-law exponent is estimated for the events whose size lies in that range. This exponent yields a fit of the distribution, and the goodness of such a fit is evaluated by means of the Kolmogorov-Smirnov (KS) test (Press et al., 1992). The purpose is to get a $p$ value, which is the probability that the KS test gives a distance between true power-law data and its fit larger than the distance obtained between the empirical data and its fit.

For instance, $p=20 \%$ would mean that truly power-law distributed data were closer than the empirical data to their respective fits in $80 \%$ of the cases, but in the rest $20 \%$ of the cases a true power law were at a larger distance than the empirical data. Therefore, in such a case the KS distance turns out to be somewhat large, but not large enough to reject that the data follow a power law with the ML exponent.

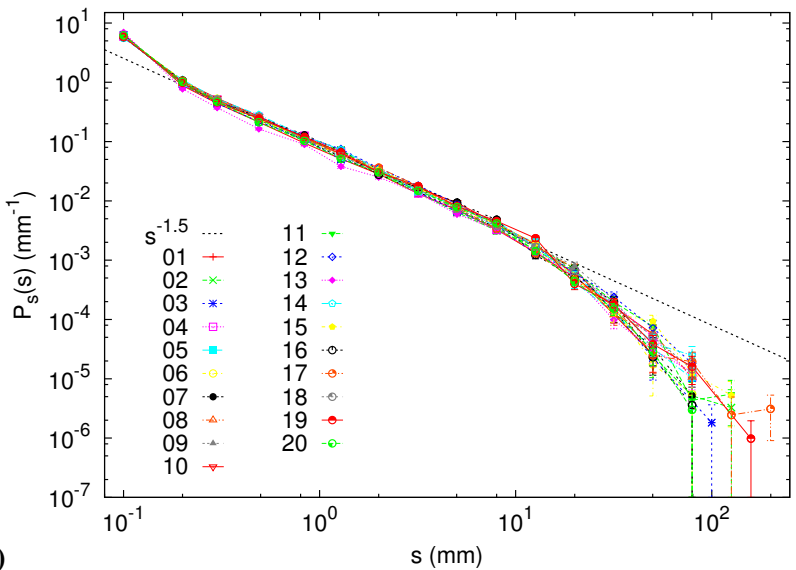

(a)
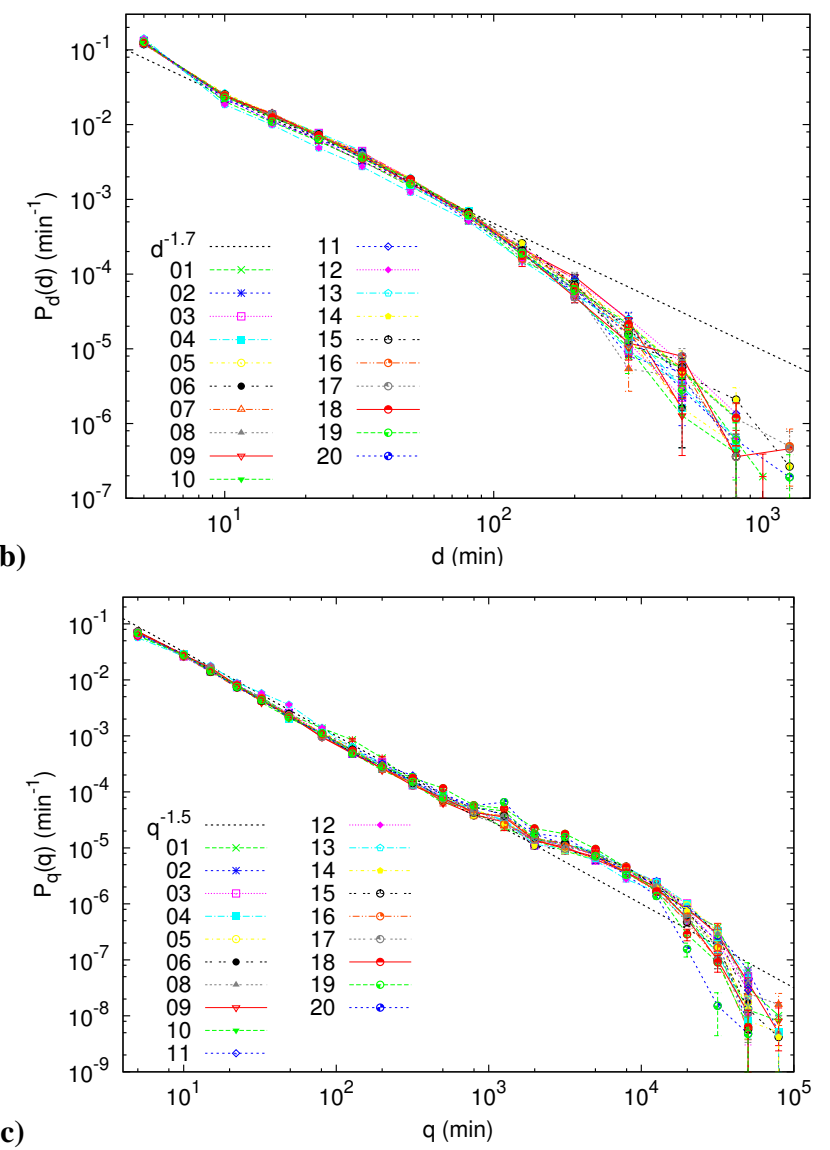

Fig. 2. Probability densities for all the sites for the whole time covered by the record of (a) event sizes, (b) event durations, and (c) dry spells.

As in the case in which some parameter is estimated from the data there is no closed formula to calculate the $p$ value, we perform Monte Carlo simulations in order to compute the statistics of the Kolmogorov-Smirnov distance and from there the $p$ value. In this way, for each $s_{\min }$ and $s_{\max }$ we get a number of data $\bar{N}_{s}$ in that range and, repeating the procedure many times, a $p$ value. We look for the values of the 
extremes $\left(s_{\min }\right.$ and $\left.s_{\max }\right)$ which maximize the number of data in between but with the restriction that the $p$ value has to be greater than $10 \%$ (this threshold is arbitrary, but the conclusions do not change if it is moved). The maximization is performed sweeping 100 values of $s_{\min }$ and 100 values of $s_{\max }$, in log-scale, in such a way that all possible ranges (within this log-resolution) are taken into account. We have to remark that, in contrast with Peters et al. (2010), we have considered always discrete probability distributions, both in the ML fit and in the simulations. Of course, it is a matter of discussion which approach (continuous or discrete) is more appropriate for discrete data that represent a continuous process. In any case, the differences in the outcomes are rather small. Notice also that the method is not based on the estimation of the probability densities shown in the previous subsections, what would be inherently more arbitrary (Clauset et al., 2009).

The results of this method are in agreement with the visual conclusions obtained in the previous subsection, as can be seen in Table 2. Starting with the size statistics, 13 out of the 20 sites yield reasonable results, with an exponent $\tau_{s}$ between 1.43 and 1.54 over a logarithmic range $s_{\max } / s_{\min }$ from 12 to more than 200. For the rest of the sites, the range is too short, less than one decade (a decade is understood from now as an order of magnitude). In the application of the algorithm, it has been necessary to restrict the value of $s_{\min }$ to be $s_{\min } \geq 0.2 \mathrm{~mm}$; otherwise, as the distributions have a concave shape (in log-scale) close to the origin (which means that there are many more events in that scale than at larger scales), the algorithm (which maximizes the number of data in a given range) prefers a short range with many data close to the origin than a larger range with less data away from the origin. It is possible that a variation of the algorithm in which the quantity that is maximized were different (for instance related with the range), would not need the restriction in the minimum size.

For the distribution of durations the resulting power laws turn out to be very limited in range; only 4 sites give not too short power laws, with $d_{\max } / d_{\min }$ from 6 to 12 and $\tau_{d}$ from 1.66 to 1.74 . The other sites yield extremely short ranges for the power law be of any relevance. The situation is analogous to the case of the distribution of sizes, but the resulting ranges are much shorter here (Peters et al., 2010). Notice that the excess of events with $d=5 \mathrm{~min}$, eliminated from the fits imposing $d_{\min } \geq 10 \mathrm{~min}$, has no counterpart in the value of the smallest rate (not shown), and therefore, we conclude that this extra number of events is due to problems in the time resolution of the data.

Considerably more satisfactory are the results for the dry spells. In all, 16 sites give consistent results, with $\tau_{q}$ from 1.45 to 1.55 in a range $q_{\max } / q_{\min }$ from 30 to almost 300 . It is noticeable that in these cases $q_{\max }$ is always below 1 day. The removal by hand of dry spells around that value should enlarge a little the power-law range. In the rest of sites, either the range is comparatively too short (for example, for the Gaià site, the power-law behavior of $P_{Q}(q)$ is interrupted at around $q=100 \mathrm{~min}$ ), or the algorithm has a tendency to include the bump the distributions show between the daily peak ( $q$ beyond $1000 \mathrm{~min}$ ) and the tail. This makes the value of the exponent smaller (around 1.25). Nevertheless, the value of the exponent is much higher than the one obtained for the equivalent problem of earthquake waiting times, where the Omori law leads to values around one, or less. This points to a fundamental differences between both kind of processes (from a statistical point of view).

In summary, the power laws for the distributions of durations are too short to be relevant, and the fits for the sizes are in the limit of what is acceptable (some cases are clear and some other not). Only the distributions of dry spells give really good power laws, with $\tau_{q}=1.50 \pm 0.05$, and for more than two decades in 6 sites.

\section{Scaling}

\subsection{Non-parametric scaling}

However, the fact that a power-law behavior does not exist over a broad range of values does not rule out the existence of scaling or SOC (Christensen and Moloney, 2005). In fact, the fulfillment of a power-law distribution in the form of Eq. (1) is only valid when finite-size effects are "small", which only happens for large enough systems. In general, when these effects are taken into account, scaling behavior leads to distributions of the form (Christensen and Moloney, 2005; Peters et al., 2010):

$P_{S}(s)=s^{-\tau_{s}} \mathcal{G}_{S}(s / s \xi)$ for $s>s_{1}$,

where $\mathcal{G}_{S}(x)$ is a scaling function that is essentially constant for $x \ll 1$ and decays fast for $x \gg 1$, accounting in this way for the finite-size effects when $s$ is above the crossover value, $s_{\xi}$; the size $s_{1}$ is just a lower cutoff limiting the validity of this description. The pure power law only emerges for $s_{\xi} \rightarrow \infty$; nevertheless, a truncated power law holds over an appreciable range if the scales given by $s_{1}$ and $s_{\xi}$ are well separated (i.e., $\left.s_{1} \ll s_{\xi}\right)$. As $s_{\xi}$ increases with system size, typically as $s_{\xi} \propto L^{D_{s}}$ (with $D_{s}$ the so-called avalanche dimension, or event-size dimension), the power-law condition (1) can only be fulfilled for large enough system sizes.

Note that, in the case of a too short power-law range or a non-conclusive fit, we still could check the existence of scaling using Eq. (2) if we knew $s \xi$ or $L$. However, $s \xi$ is difficult to measure, needing a parameterization of the scaling function, and it is not clear what the system size $L$ is for rainfall. It could be the vertical extension of the clouds, or the depth of the troposphere. Nevertheless, it is important to realize that the scaling ansatz (2) still can be checked from data without knowledge of $L$ or $s \xi$. First, notice that the ansatz implies that the $k$-order moment of $s$ scales with $L$ as

$\left\langle s^{k}\right\rangle \propto L^{D_{s}\left(k+1-\tau_{s}\right)}$ for $1<\tau_{s}<k+1$, 
Table 2. Results of the power-law fitting and goodness-of-fit tests applied to event sizes, event durations, and dry-spell durations (in mm or in $\min$ ), for the period of nine and a half years specified in the main text. The table displays the minimum of the fitting range, $s_{\text {min }}$, and the ratio between the maximum and the minimum of the fitting range (logarithmic range, $s_{\max } / s_{\min }$ ), total number of events, number of events in fitting range $\left(\bar{N}_{s}, \bar{N}_{d}\right.$, and $\bar{N}_{q}$, for $s, d$, and $q$, respectively), and the power-law exponent with its uncertainty (one standard deviation) calculated as stated by Bauke (2007) and displayed between parenthesis as the variation of the last digit.

\begin{tabular}{lcrcccccccrrrrc}
\hline Site & $s_{\min }$ & $\frac{s_{\max }}{s_{\min }}$ & \multicolumn{1}{c}{$N_{s}$} & $\bar{N}_{s}$ & $\tau_{s}$ & $d_{\min }$ & $\frac{d_{\max }}{d_{\min }}$ & $\bar{N}_{d}$ & $\tau_{d}$ & $q_{\min }$ & $\frac{q_{\max }}{q_{\min }}$ & $N_{q}$ & $\bar{N}_{q}$ & $\tau_{q}$ \\
\hline 1 & 0.2 & 180.5 & 5393 & 1886 & $1.54(2)$ & 10 & 10.0 & 1668 & $1.67(4)$ & 95 & 7.8 & 5387 & 743 & $1.75(7)$ \\
2 & 0.2 & 4.5 & 5236 & 1323 & $1.64(6)$ & 10 & 4.0 & 1581 & $1.60(6)$ & 5 & 273.0 & 5231 & 4729 & $1.46(1)$ \\
3 & 0.2 & 155.5 & 5749 & 2111 & $1.53(2)$ & 10 & 6.0 & 1726 & $1.66(5)$ & 10 & 80.0 & 5745 & 3207 & $1.53(2)$ \\
4 & 0.2 & 12.0 & 5108 & 1745 & $1.43(3)$ & 10 & 3.5 & 1564 & $1.41(7)$ & 5 & 196.0 & 5103 & 4520 & $1.47(1)$ \\
5 & 0.2 & 140.0 & 5289 & 2106 & $1.52(2)$ & 10 & 3.5 & 1530 & $1.58(7)$ & 20 & 47.3 & 5287 & 1706 & $1.45(2)$ \\
6 & 0.2 & 68.0 & 4924 & 1969 & $1.49(2)$ & 10 & 3.5 & 1441 & $1.59(7)$ & 20 & 31.3 & 4926 & 1537 & $1.47(3)$ \\
7 & 0.2 & 105.5 & 5219 & 2234 & $1.51(2)$ & 10 & 3.5 & 1621 & $1.51(7)$ & 5 & 256.0 & 5215 & 4734 & $1.51(1)$ \\
8 & 0.2 & 213.0 & 5112 & 2047 & $1.53(2)$ & 10 & 4.0 & 1567 & $1.55(6)$ & 15 & 65.0 & 5107 & 2098 & $1.50(2)$ \\
9 & 0.2 & 5.0 & 5366 & 1459 & $1.53(5)$ & 10 & 4.0 & 1658 & $1.51(6)$ & 10 & 90.0 & 5419 & 2889 & $1.55(2)$ \\
10 & 0.2 & 19.0 & 6691 & 2452 & $1.51(2)$ & 10 & 3.5 & 2066 & $1.57(6)$ & 25 & 33.0 & 6685 & 1758 & $1.48(3)$ \\
11 & 0.2 & 65.0 & 6224 & 2373 & $1.49(2)$ & 10 & 5.0 & 1932 & $1.56(5)$ & 45 & 468.3 & 6219 & 2005 & $1.24(1)$ \\
12 & 0.2 & 4.0 & 5967 & 1500 & $1.53(6)$ & 10 & 4.0 & 1889 & $1.49(6)$ & 5 & 235.0 & 5961 & 5376 & $1.47(1)$ \\
13 & 0.3 & 66.7 & 8330 & 1853 & $1.45(2)$ & 10 & 12.5 & 2288 & $1.74(3)$ & 130 & 158.5 & 8328 & 1501 & $1.27(2)$ \\
14 & 0.2 & 3.5 & 6525 & 1711 & $1.56(6)$ & 10 & 5.0 & 2299 & $1.62(4)$ & 5 & 215.0 & 6520 & 5906 & $1.47(1)$ \\
15 & 0.3 & 3.7 & 6485 & 1102 & $1.39(7)$ & 10 & 5.0 & 2095 & $1.64(5)$ & 15 & 49.7 & 6479 & 2560 & $1.51(2)$ \\
16 & 0.2 & 3.5 & 7491 & 1852 & $1.59(5)$ & 10 & 4.0 & 2385 & $1.59(5)$ & 5 & 214.0 & 7510 & 6789 & $1.50(1)$ \\
17 & 0.2 & 80.5 & 6962 & 2853 & $1.52(2)$ & 10 & 3.5 & 2087 & $1.60(6)$ & 10 & 68.5 & 6958 & 3719 & $1.52(1)$ \\
18 & 0.2 & 41.5 & 7511 & 2847 & $1.51(2)$ & 10 & 3.5 & 2238 & $1.57(6)$ & 20 & 31.0 & 7507 & 2302 & $1.53(2)$ \\
19 & 0.2 & 99.5 & 6767 & 2742 & $1.47(2)$ & 10 & 3.5 & 1958 & $1.60(6)$ & 50 & 21.7 & 6800 & 1378 & $1.26(3)$ \\
20 & 0.2 & 3.5 & 9012 & 2047 & $1.69(5)$ & 10 & 8.5 & 2972 & $1.66(3)$ & 15 & 34.3 & 9007 & 3367 & $1.50(2)$ \\
\hline
\end{tabular}

if $s_{1} \ll s_{\xi}$, see Christensen and Moloney (2005). Second, Eq. (2) can be written in a slightly different form, as a scaling law,

$P_{S}(s)=L^{-D_{s} \tau_{s}} \mathcal{F}_{S}\left(s / L^{D_{s}}\right)$ for $s>s_{1}$,

where the new scaling function $\mathcal{F}_{S}(x)$ is defined as $\mathcal{F}_{S}(x) \equiv$ $x^{-\tau_{S}} \mathcal{G}_{S}(x / a)$ ( $a$ is the constant of proportionality between $s_{\xi}$ and $L^{D_{s}}$ ). This form of $P_{S}(s)$ (in fact, $P_{S}(s, L)$ ), with an arbitrary $\mathcal{F}_{S}$, is the well-known scale-invariance condition for functions with two variables (Christensen and Moloney, 2005). Changes of scale (linear transformations) in $s$ and $L$ may leave the shape of the function $P_{S}(s, L)$ unchanged (this is what scale invariance really means, power laws are just a particular case in one dimension).

Substituting $L^{D_{s}} \propto\left\langle s^{2}\right\rangle /\langle s\rangle$ and $L^{D_{s} \tau_{s}} \propto L^{2 D_{s}} /\langle s\rangle \propto$ $\left\langle s^{2}\right\rangle^{2} /\langle s\rangle^{3}$ (from the scaling of $\left\langle s^{k}\right\rangle$, assuming $\tau_{s}<2$ ) into Eq. (4) leads to

$P_{S}(s)=\langle s\rangle^{3}\left\langle s^{2}\right\rangle^{-2} \tilde{\mathcal{F}}_{\mathcal{S}}\left(s\langle s\rangle /\left\langle s^{2}\right\rangle\right)$,

where $\tilde{\mathcal{F}}_{\mathcal{S}}(x)$ is essentially the scaling function $\mathcal{F}_{S}(x)$, absorbing the proportionality constants. Therefore, if scaling holds, a plot of $\left\langle s^{2}\right\rangle^{2} P_{S}(s) /\langle s\rangle^{3}$ versus $s\langle s\rangle /\left\langle s^{2}\right\rangle$ for all the sites has to yield a collapse of the distributions into a single curve, which draws $\tilde{\mathcal{F}}_{\mathcal{S}}(x)$ (a similar procedure is outlined in Rosso et al., 2009). In order to proceed, the mean and the quadratic mean, $\langle s\rangle$ and $\left\langle s^{2}\right\rangle$, can be easily estimated from data. Since no estimation of parameters is involved for this procedure, we call it non-parametric scaling.

The outcome for $P_{S}(s), P_{\mathrm{D}}(d)$, and $P_{Q}(q)$ is shown in Fig. 3a, b, and c, with reasonable results, especially for the distribution of dry spells. The plot suggests that the scaling function $\mathcal{G}_{Q}$ of the dry-spell distribution has a maximum around $x \simeq 1$, but this is not in disagreement with our approach, which only assumed a constant scaling function for small $x$ and a fast decay for large $x$.

Note that the quotient $\left\langle s^{2}\right\rangle /\langle s\rangle$ gives the scale for the crossover value $s_{\xi}$ (as $s_{\xi} \propto\left\langle s^{2}\right\rangle /\langle s\rangle$, with a constant of proportionality that depends on the scaling function $\mathcal{G}_{S}$ and on $\left.s_{1} / s_{\xi}\right)$, and therefore it is the ratio of the second moment to the mean and not the mean which describes the scaling behavior of the distribution. This can have important implications for extreme events: an increase in the value of the mean is not proportional to an increase of the most extreme events, represented by $s_{\xi}$. For the case of event sizes, we get values of $\left\langle s^{2}\right\rangle /\langle s\rangle$ between 10 and $30 \mathrm{~mm}$ (which is a variability much higher than that of $\langle s\rangle)$, and therefore the condition $s_{1} \ll s_{\xi}$ is very well fulfilled (assuming that the moment ratio $\left\langle s^{2}\right\rangle /\langle s\rangle$ is of the same order as $s_{\xi}$, and with $s_{1} \simeq s_{\min }$ ), which is a test for the consistency of our approach. For dry spells $\left\langle q^{2}\right\rangle /\langle q\rangle$ is between 5 and 13 days, which is even better for the applicability of the scaling analysis. The case of the event durations is somewhat "critical", with $\left\langle d^{2}\right\rangle /\langle d\rangle$ between 70 


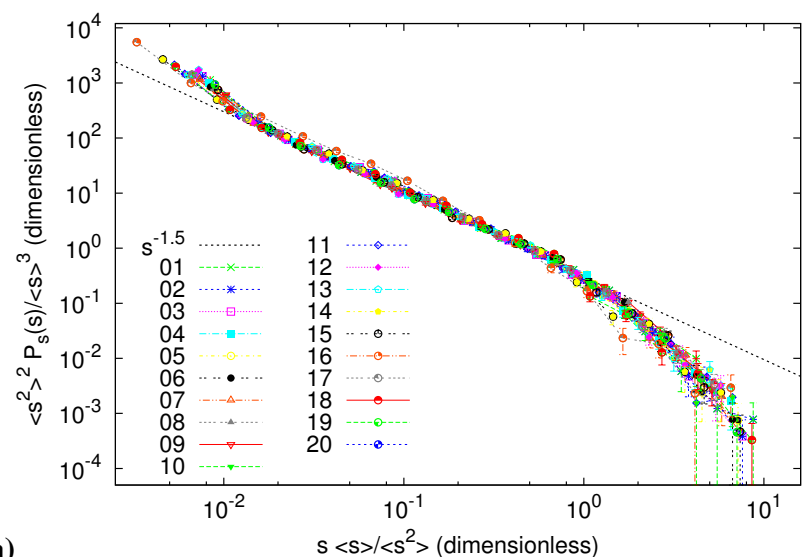

(a)

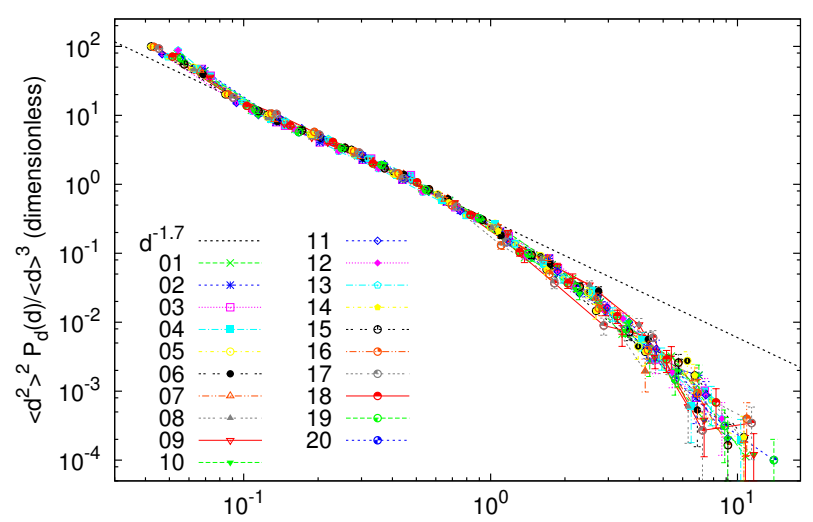

(b)

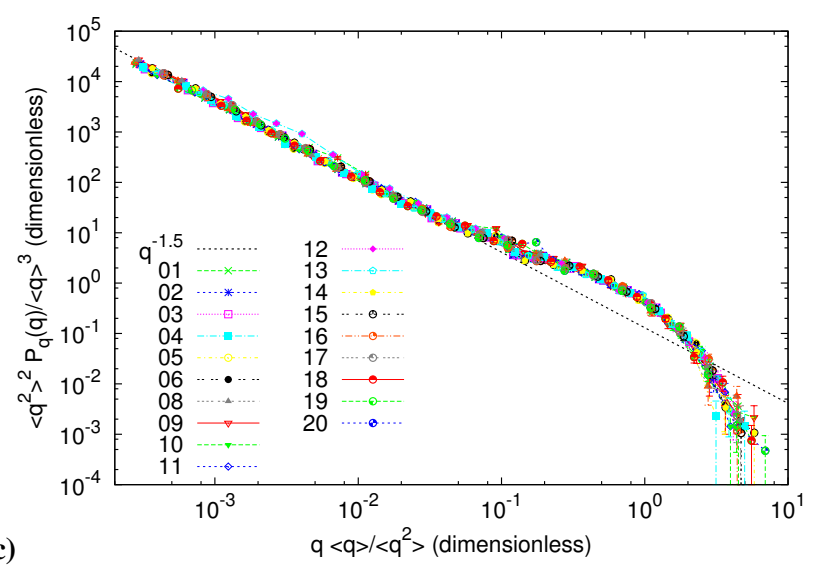

Fig. 3. Collapse of the probability densities for all the sites for the whole time covered by the record of: (a) event sizes, (b) event durations, and (c) dry spells. Rescaling is performed using the first and second moment of each distribution, following Eq. (5).

and $120 \mathrm{~min}$, which yields $d_{\xi} / d_{1}$ in the range from 14 to 24 . Nevertheless, we observe that the condition $s_{1} \ll s_{\xi}$ for the power law to show up is stronger than the same condition for the scaling analysis to be valid.

\subsection{Parametric scaling}

Further, a scaling ansatz as Eq. (2) or (4) allows an estimation of the exponent $\tau_{s}$, even in the case in which a power law cannot be fit to the data. From the scaling of the moments of $s$ we get, taking $k=1, L^{D_{s}} \propto\langle s\rangle^{1 /\left(2-\tau_{s}\right)}$ and $L^{D_{s} \tau_{s}} \propto\langle s\rangle^{\tau_{s} /\left(2-\tau_{s}\right)}$ (again with $\tau_{s}<2$ ); therefore, substituting into Eq. (4),

$P_{S}(s)=\langle s\rangle^{-\tau_{s} /\left(2-\tau_{s}\right)} \hat{\mathcal{F}}_{\mathcal{S}}\left(s /\langle s\rangle^{1 /\left(2-\tau_{s}\right)}\right)$.

One only needs to find the value of $\tau_{s}$ that optimizes the collapse of all the distributions, that is, that makes the previous equation valid, or at least as close to validity as possible. As the scaling depends on the parameter $\tau_{s}$, we refer to this procedure as parametric scaling.

We therefore need a measurement to quantify distance between rescaled distributions. In order to do that, we have chosen to work with the cumulative distribution function, $R_{S}(s) \equiv \int_{s}^{\infty} P_{S}\left(s^{\prime}\right) d s^{\prime}$, rather than with the density (to be rigorous, $R_{S}(s)$ is the complementary of the cumulative distribution function, and is called survivor function or reliability function in some contexts). Although in practice both $P_{S}(s)$ and $R_{S}(s)$ contain the same probabilistic information, the reason to work with $R_{S}(s)$ is double: the estimation of the cumulative distribution function does not depend of an arbitrarily selected bin width $d s$ (Press et al., 1992), and it does not give equal weight to all scales in the representation of the function (i.e., in the number of points that constitute the function). The scaling laws (4) and (6) turn out to be, therefore,

$R_{S}(s)=L^{-D_{s}\left(\tau_{s}-1\right)} \mathcal{H}_{S}\left(s / L^{D_{s}}\right)$

$R_{S}(s)=\langle s\rangle^{-\left(\tau_{s}-1\right) /\left(2-\tau_{s}\right)} \hat{\mathcal{H}}_{\mathcal{S}}\left(s /\langle s\rangle^{1 /\left(2-\tau_{s}\right)}\right)$,

where $\mathcal{H}_{S}(x)$ and $\hat{\mathcal{H}}_{\mathcal{S}}(x)$ are the corresponding scaling functions.

The first step of the method of collapse is to merge all the pairs $\left\{s, R_{S}(s)\right\}_{i}$ into a unique rescaled function $\{x, y\}$. If $i=1, \ldots, 20$ runs for all sites, and $j=1, \ldots, M_{s}(i)$ for all the different values that the size of events takes on site $i$ (note that $\left.M_{S}(i) \leq N_{s}(i)\right)$,

$$
\begin{aligned}
& x_{\ell}(\tau) \equiv \log \left(s_{j i} /\langle s\rangle_{i}^{1 /(2-\tau)}\right), \\
& y_{\ell}(\tau) \equiv \log \left(R_{i}\left(s_{j i}\right)\langle s\rangle_{i}^{(\tau-1) /(2-\tau)}\right),
\end{aligned}
$$

where $s_{j i}$ is the $j$ th value of the size in site $i,\langle s\rangle_{i}$ the mean on $s$ in $i, R_{i}\left(s_{j i}\right)$ the cumulative distribution function in $i$, and $\tau$ a possible value of the exponent $\tau_{s}$. The index $\ell$ labels the new function, from 1 to $\sum_{\forall i} M_{s}(i)$, in such a way that $x_{\ell}(\tau) \leq x_{\ell+1}(\tau)$; this means that the pairs $x_{\ell}(\tau), y_{\ell}(\tau)$ are sorted by increasing $x$.

Then, we just compute

$$
D(\tau) \equiv \sum_{\forall \ell}\left(\left[x_{\ell}(\tau)-x_{\ell+1}(\tau)\right]^{2}+\left[y_{\ell}(\tau)-y_{\ell+1}(\tau)\right]^{2}\right),
$$


which represents the sum of all Euclidean distances between the neighboring points in a (tentative) collapse plot in logarithmic scale. The value of $\tau$ which minimizes this function is identified with the exponent $\tau_{s}$ in Eq. (2). We have tested the algorithm applying it to SOC models whose exponents are well known (not shown).

The results of this method applied to our data sets, not only for the size distributions but also to the distributions of $d$, are highly satisfactory. There is only one requirement: the removal of the first point in each distribution $(s=0.1 \mathrm{~mm}$ and $d=5 \mathrm{~min}$ ), as with the ML fits. The exponents we find are $\tau_{s}=1.52 \pm 0.12$ and $\tau_{d}=1.69 \pm 0.01$, in agreement with the ones obtained by the power-law fitting method presented above; the corresponding rescaled plots are shown in Fig. 4. Although the visual display does not allow to evaluate properly the quality of the collapse, the reduction in the value of the function $D(\tau)$ is notable. Then, the performance of the method is noteworthy, taking into account that the mean values of the distributions show little variation in most cases. In addition, the shape of the scaling function $\mathcal{G}_{S}$ can be obtained by plotting, as suggested by Eq. (2), $s^{\tau_{s}} P_{S}(s)$ versus $s /\langle s\rangle^{1 /\left(2-\tau_{s}\right)}$, and the same for the other variable, $d$. Figure 5 displays what is obtained for each distribution. In contrast, the application of this method to $P_{Q}(q)$ does not yield consistent results, as $\tau_{q}$ turns out to be rather small (1.24). Notice that the existence of a daily peak in the distributions is an obstacle to a data collapse, as the peak prevents a good scaling.

\section{Discussion and conclusions}

We have performed an in-depth study of the properties of rain-event observables in rainfall in the Mediterranean region in order to check if power-law distributions and scale invariance can be useful for modeling rain events and dry spells. The results support this hypothesis, which had not been checked before in this region or for this kind of data resolution. For the distributions of rain-event sizes, we get power-law exponents valid for one or two decades in the majority of sites, with exponent values $\tau_{s} \simeq 1.50 \pm 0.05$. For the distributions of event durations, the fitting ranges are shorter, reaching in the best case one decade, with exponents $\tau_{d} \simeq 1.70 \pm 0.05$. This range is expected to be shorter than for event sizes, given that these combine the event duration distribution with the rain rate (Peters et al., 2010). And finally, the dry spell distributions yield the more notable power-law fits, with exponents in the range $\tau_{q} \simeq 1.50 \pm 0.05$, in some cases for more than 2 decades.

These results are compatible with the ones obtained for the Baltic sea by Peters et al. (2002), which yielded $\tau_{s} \simeq$ $\tau_{q} \simeq 1.4$ and $\tau_{d} \simeq 1.6$. The agreement is remarkable, taking into account the different nature of the data analyzed and the disparate fitting procedures. However, the concordance with the more recent results of Peters et al. (2010) is not very good, quantitatively. That previous study, with a
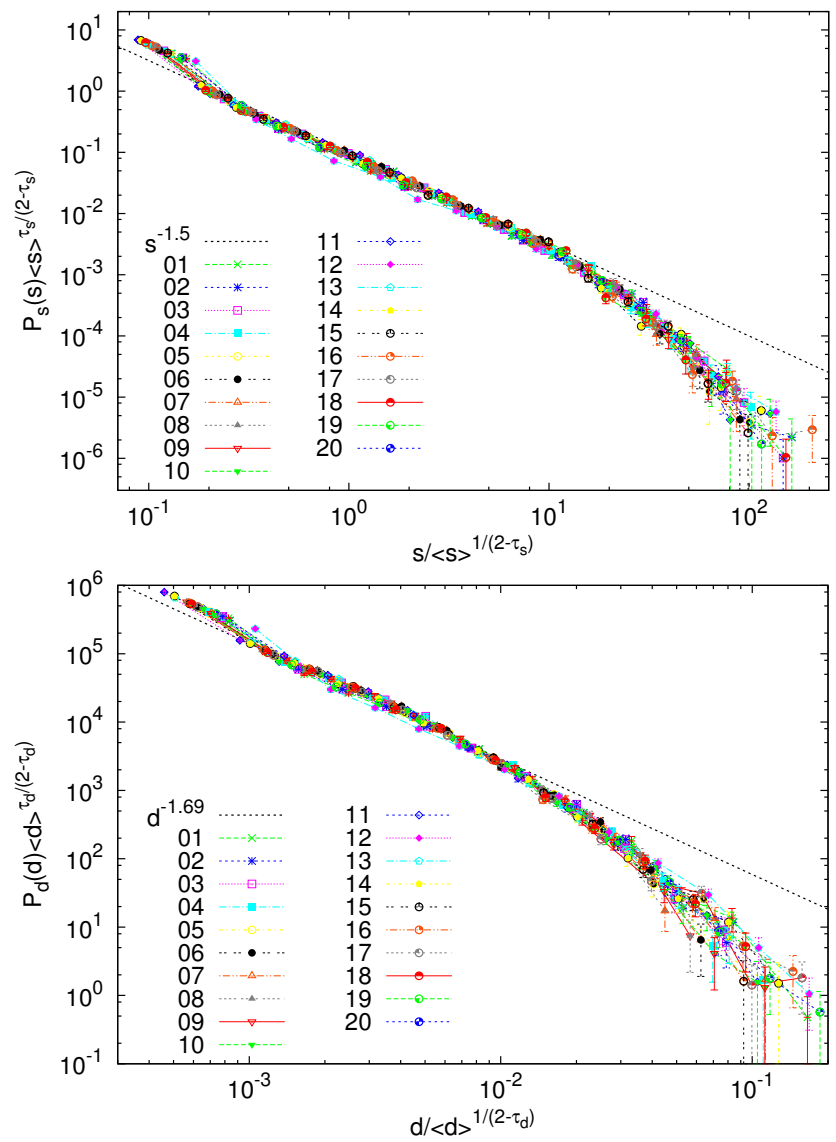

Fig. 4. Collapse of the probability densities for all the sites for the whole time covered by the record of (a) event sizes and (b) event durations; rescaled using Eq. (6) with the exponents: $\tau_{s}=1.52$ and $\tau_{d}=1.69$, determined minimizing the Euclidean distance between parametrically collapsed distributions. Units are $\mathrm{mm}$ or $\min$ to the corresponding powers appearing in the axes.

minimum detection rate of $0.2 \mathrm{mmh}^{-1}$ and a time resolution $\Delta t=1 \mathrm{~min}$, found $\tau_{s} \simeq 1.17$ for several sites across different climates, using essentially the same statistical techniques as in the present study. For dry-spell durations the exponents were close in many cases to $\tau_{q} \simeq 1$.3. The difference between the value of the exponents may be due to data resolution (but then, our agreement with the values in the first paper may be fortuitous). Changes in the detection threshold have a nontrivial repercussion in the size and duration of the events and the dry spells (an increase in the threshold can split one single event into two or more separate ones but also can remove events). Further, better time resolution and lower detection threshold allow the detection of smaller events, enlarging the power-law range and reducing the weight from the part close to the crossover point (where the distribution becomes steeper); this trivially leads to smaller values of the exponent. In the dry-spell case, the power-law range in this study is enough to guarantee that our estimation of the exponents are robust, so the discrepancy with Peters et al. (2010) may 

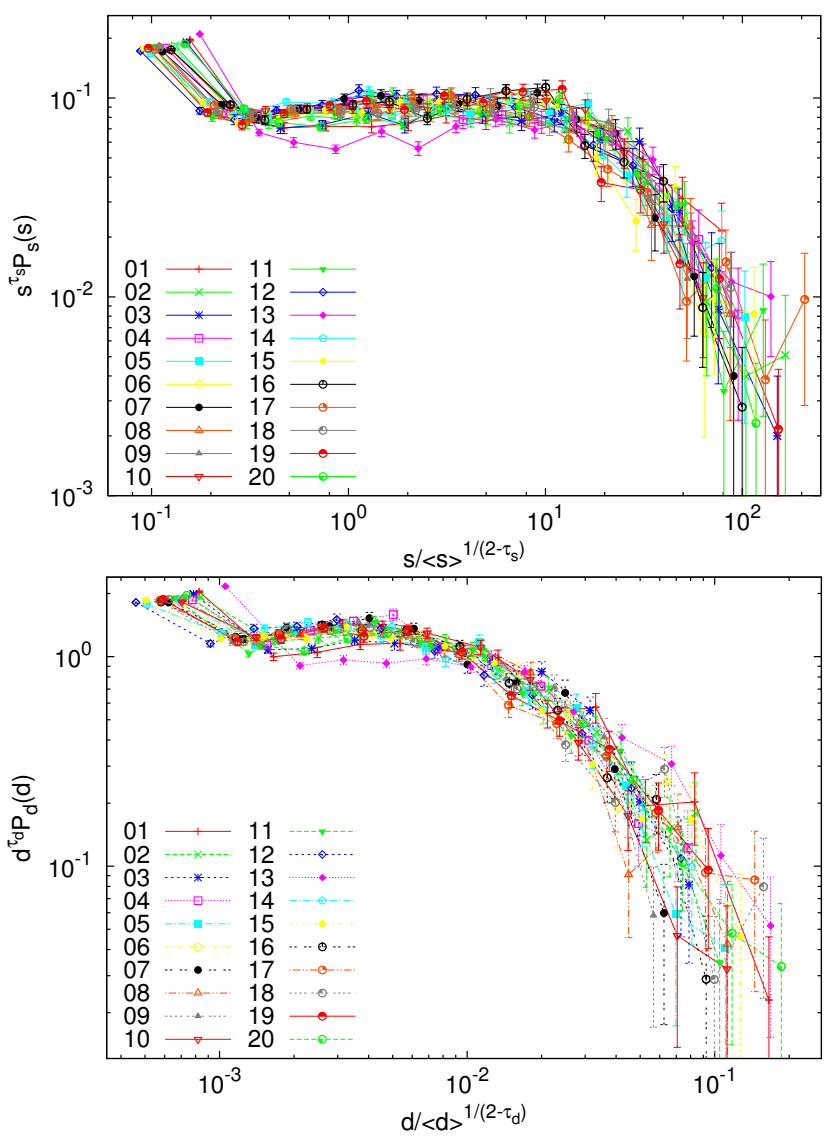

Fig. 5. Inferred scaling functions $\mathcal{G}_{S}$ and $\mathcal{G}_{\mathrm{D}}$ (except for a proportionality factor in the $x$ axis) corresponding to the rescaled distributions of $s$ and $d$ in Fig. 4, multiplied by $s^{\tau_{s}}$ and $d^{\tau_{d}}$. Units in the abscissae are as in the previous plot, whereas in the ordinates these are $\mathrm{mm}^{\tau_{s}-1}$ and $\min ^{\tau_{d}-1}$.

be due to the non-trivial effect of the change in the detection thresholds or differences on the measurement devices.

On the other hand, finite size effects can explain the limited power-law range obtained for $s$ and $d$ observables, as it occurs in (self-organized and non-self-organized) critical phenomena. The finite-size analysis performed, in terms of combinations of powers of the moments of the distributions, supports this conclusion. The collapse of the distributions is a clear signature of scale invariance: different sites share a common shape of the rain-event and dry-spell distributions, with differences in the scale of those distributions, depending on system size. Then, in the ideal case of an infinite system, the power laws would lack an upper cutoff. Moreover, the collapse of the distributions allows an independent estimate of the power-law exponents, which, for event durations and sizes, are in surprising agreement with the values obtained by the maximum-likelihood fit. For dry spell durations, a daily peak in the distributions hinders their collapse.
Nevertheless, future work should consider spatially extended events. Our measurements are taken in a point of the surface which reflects information on the vertical scale, then, the results could be affected by this. Another related but different issue are the correlations between the sites, which have not been taken into account. Further, in order to establish a link with SOC or not, one would need to measure, simultaneously, other variables, such as water vapor content (Peters and Neelin, 2006). Our results show that these studies can be undertaken without high-resolution rain data.

The implications of the results for hazard assessment are also of great interest: if there is not a characteristic rain-event size, then there is neither a definite separation nor a fundamental difference between the smallest harmless rains and the most hazardous storms. Further, it is generally believed that the critical evolution of events in SOC systems implies that, at a given instant, it is equally likely that the event intensifies or weakens, which would make detailed prediction unattainable. However, this view has been recently proved wrong, as it has been reported that a critical evolution describes the dynamics of some SOC systems only on average; further, the existence of finite size effects can be used for prediction (Garber et al., 2009; Martin et al., 2010). Interestingly, in the case of rain, it has been recently shown by Molini et al. (2011) that knowledge of internal variables of the system allows some degree of prediction for the duration of the events, related also to the departure of the system from quasi-equilibrium conditions.

Finally, we urge studies which explore the effects of resolution and detection-threshold value in high-resolution rain data. A common misbelief in the SOC approach is that avalanches happen following a memoryless process, leading therefore to exponential distributions for the waiting times (Corral, 2005). This has been refuted if a threshold on the intensity is present (Paczuski et al., 2005). In this case, times between avalanches follow a power-law distribution, as we find for dry spells.

In summary, we conclude that the statistics of rainfall events in the NW Mediterranean area studied are in agreement with the SOC-paradigm expectations but also with any other mechanism for power-law genesis. This is the first time this study is realized for this region and it is a confirmation of what has been found for other places of the world, but in our case using data with lower resolution.

Acknowledgements. This work would not have been possible without the collaboration of the Agència Catalana de l'Aigua (ACA), which generously shared its rain data with us. We have benefited a lot from a long-term collaboration with Ole Peters, and are grateful also to R. Romero and A. Rosso, for addressing us towards the ACA data and an important reference (Rosso et al., 2009), and to J. E. Llebot for providing support and encouragement. We also thank Joaquim Farguell from ACA. A. Mugnai and other people of the Plinius conferences made us realize of the importance of our results for the non-linear geophysics community. A. Deluca 
enjoyed a Ph.D. grant of the Centre de Recerca Matemàtica. Spanish grants related to this work are FIS2009-09508, 2009SGR-164, and FIS2012-31324.

Edited by: D. Schertzer

Reviewed by: two anonymous referees

\section{References}

ACA (Agència Catalana de l'Aigua/Catalan Water Agency), available at: http://aca-web.gencat.cat/aca (last access: April 2014), 2014

Andrade, R. F. S., Schellnhuber, H. J., and Claussen, M.: Analysis of rainfall records: possible relation to self-organized criticality, Physica A, 254, 557-568, 1998.

Bak, P.: How Nature Works: The Science of Self-Organized Criticality, Copernicus, New York, 1996.

Bauke, H.: Parameter estimation for power-law distributions by maximum likelihood methods, Eur. Phys. J. B, 58, 167-173, 2007.

Bodenschatz, E., Malinowski, S., Shaw, R., and Stratmann, F.: Can we understand clouds without turbulence?, Science, 327, 970971,2010

Cahalan, R. and Joseph, J.: Fractal statistics of cloud fields, Mon. Weather Rev., 117, 261-272, 1989.

Christensen, K. and Moloney, N. R.: Complexity and Criticality, Imperial College Press, London, 2005.

Clauset, A., Shalizi, C. R., and Newman, M. E. J.: Power-law distributions in empirical data, SIAM Rev., 51, 661-703, 2009.

Corral, Á.: Comment on "Do Earthquakes Exhibit SelfOrganized Criticality?”, Phys. Rev. Lett., 95, 159801, doi:10.1103/PhysRevLett.95.159801,2005.

Corral, A., Font, F., and Camacho, J.: Noncharacteristic halflives in radioactive decay, Phys. Rev. E, 83, 066103, doi:10.1103/PhysRevE.83.066103, 2011.

Deidda, R.: Rainfall downscaling in a space-time multifractal framework, Water Resour. Res., 36, 1779-1794, doi:10.1029/2000WR900038, 2000.

Deluca, A. and Corral, A.: Fitting and goodness-of-fit test of nontruncated and truncated power-law distributions, Acta Geophys., 61, 1351-1394, 2013.

Dickman, R.: Rain, Power Laws, and Advection, Phys. Rev. Lett., 90, 108701, doi:10.1103/PhysRevLett.90.108701, 2003.

Dickman, R., Vespignani, A., and Zapperi, S.: Self-organized criticality as an absorbing-state phase transition, Phys. Rev. E, 57, 5095-5105, 1998.

Dickman, R., Muñoz, M. A., Vespignani, A., and Zapperi, S.: Paths to Self-Organized Criticality, Braz. J. Phys., 30, 27-41, 2000.

Garber, A., Hallerberg, S., and Kantz, H.: Predicting extreme avalanches in self-organized critical sandpiles, Phys. Rev. E, 80, 026124, doi:10.1103/PhysRevE.80.026124, 2009.

Hergarten, S.: Self-Organized Criticality in Earth Systems, Springer, Berlin, 2002.

Hooge, C., Lovejoy, S., Schertzer, D., Pecknold, S., Malouin, J.-F., and Schmitt, F.: Mulifractal phase transitions: the origin of selforganized criticality in earthquakes, Nonlin. Processes Geophys., 1, 191-197, doi:10.5194/npg-1-191-1994, 1994.

Jensen, H. J.: Self-Organized Criticality, Cambridge University Press, Cambridge, 1998.
Lavergnat, J. and Golé, P.: A stochastic model of raindrop release: Application to the simulation of point rain observations, J. Hydrol., 328, 8-19, 2006.

Lovejoy, S.: Area-perimeter relation for rain and cloud areas, Science, 216, 185-187, 1982.

Lovejoy, S. and Schertzer, D.: Multifractals and rain, in: New Uncertainty Concepts in Hydrology and Water Resources, edited by: Kundzewicz, Z. W., Cambridge University Press, 61-103, 1995.

Lovejoy, S. and Schertzer, D.: The weather and climate, Cambridge University Press, Cambridge, 2013.

Malamud, B. D.: Tails of natural hazards, Phys. World, 17, 31-35, 2004.

Malamud, B. D., Morein, G., and Turcotte, D. L.: Forest Fires: An Example of Self-Organized Critical Behavior, Science, 281, 1840-1842, 1998.

Martin, E., Shreim, A., and Paczuski, M.: Activity-dependent branching ratios in stocks, solar x-ray flux, and the BakTang-Wiesenfeld sandpile model, Phys. Rev. E, 81, 016109 , doi:10.1103/PhysRevE.81.016109, 2010.

Molini, L., Parodi, A., Rebora, N., and Craig, G. C.: Classifying severe rainfall events over Italy by hydrometeorological and dynamical criteria, Q. J. Roy. Meteor. Soc., 137, 148-154, 2011.

Olsson, J., Niemczynowicz, J., and Berndtsson, R.: Fractal analysis of high-resolution rainfall time series, J. Geophys. Res., 98, 23265-23274, 1993.

OTT (OTT Hydromet GmbH), available at: http://www. ott-hydrometry.de (last access: April 2014), 2014.

Paczuski, M., Boettcher, S., and Baiesi, M.: Interoccurrence times in the Bak-Tang-Wiesenfeld sandpile model: A comparison with the observed statistics of solar flares, Phys. Rev. Lett., 95, 181102, doi:10.1103/PhysRevLett.95.181102, 2005.

Peters, O. and Christensen, K.: Rain: Relaxations in the sky, Phys. Rev. E, 66, 036120, doi:10.1103/PhysRevE.66.036120, 2002.

Peters, O. and Christensen, K.: Rain viewed as relaxational events, J. Hydrol., 328, 46-55, 2006.

Peters, O. and Neelin, J. D.: Critical phenomena in atmospheric precipitation, Nat. Phys., 2, 393-396, 2006.

Peters, O., Hertlein, C., and Christensen, K.: A Complexity View of Rainfall, Phys. Rev. Lett., 88, 018701, doi:10.1103/PhysRevLett.88.018701, 2002.

Peters, O., Neelin, J., and Nesbitt, S.: Mesoscale convective systems and critical clusters, J. Atmos. Sci., 66, 2913-2924, 2009.

Peters, O., Deluca, A., Corral, A., Neelin, J. D., and Holloway, C. E.: Universality of rain event size distributions, J. Stat. Mech.Theory E., P11030, doi:10.1088/1742-5468/2010/11/P11030, 2010.

Press, W. H., Teukolsky, S. A., Vetterling, W. T., and Flannery, B. P.: Numerical Recipes in FORTRAN, 2nd Edn., Cambridge University Press, Cambridge, 1992.

Rosso, A., Le Doussal, P., and Wiese, K. J.: Avalanche-size distribution at the depinning transition: A numerical test of the theory, Phys. Rev. B, 80, 144204, doi:10.1103/PhysRevB.80.144204, 2009.

Schertzer, D. and Lovejoy, S.: Hard and soft multifractal processes, Physica A, 185, 187-194, 1992.

Schertzer, D. and Lovejoy, S.: Multifractal generation of selforganized criticality, in: Proceedings of the Second IFIP Working Conference on Fractals in the Natural and Applied Sciences, 
edited by: Novak, M. M., Elsevier, North-Holland: Amsterdam, 325-339, 1994.

Schertzer, D. and Lovejoy, S.: Multifractals, generalized scale invariance and complexity in geophysics, Int. J. Bifurcat. Chaos, 21, 3417-3456, 2011.

Sornette, A. and Sornette, D.: Self-organized Criticality and Earthquakes, Europhys. Lett., 9, 197-202, 1989.

Sornette, D.: Critical Phenomena in Natural Sciences, 2nd Edn., Springer, Berlin, 2004.

Tang, C. and Bak, P.: Critical Exponents and Scaling Relations for Self-Organized Critical Phenomena, Phys. Rev. Lett., 60, 23472350,1988 .
Telesca, L., Lapenna, V., Scalcione, E., and Summa, D.: Searching for time-scaling features in rainfall sequences, Chaos Soliton. Fract., 32, 35-41, 2007.

Veneziano, D. and Lepore, C.: The scaling of temporal rainfall, Water Resour. Res., 48, W08516, doi:10.1029/2012WR012105, 2012.

von Mises, R.: Mathematical Theory of Probability and Statistics, Academic Press, New York, 1964.

Wood, R. and Field, P.: The distribution of cloud horizontal sizes, J. Climate, 24, 4800-4816, 2011. 


\section{Appendix A}

\section{Details on the estimation of the probability density}

In practice, the estimation of the density from data is performed taking a value of $d s$ large enough to guarantee statistical significance, and then compute $P_{S}(s)$ as $n(s) /\left(N_{s} \Delta\right)$, where $n(s)$ is the number of events with size in the range between $s$ and $s+d s, N_{s}$ the total number of events, and $\Delta$ is defined as

$\Delta=\mathcal{R}_{s}\left(\left\lfloor(s+d s) / \mathcal{R}_{s}\right\rfloor-\left\lfloor s / \mathcal{R}_{s}\right\rfloor\right)$,

where $\lfloor x\rfloor$ is the integer part of $x$ and $\mathcal{R}_{s}$ the resolution of $s$, that is, $\mathcal{R}_{s}=0.1 \mathrm{~mm}$ (but note that high resolution means low $\mathcal{R}_{s}$ ). Therefore, $\Delta / \mathcal{R}_{s}$ is the number of possible different values of the variable in the interval considered. Notice that using $\Delta$ instead of $d s$ in the denominator of the estimation of $P_{S}(s)$ allows one to take into account the discreteness of $s$. If $\mathcal{R}_{s}$ tended to zero, then $\Delta \rightarrow d s$ and the discreteness effects would become irrelevant.

How large does $d s$ have to be to guarantee the statistical significance of the estimation of $P_{S}(s)$ ? Working with long-tailed distributions (where the variable covers a broad range of scales) a very useful procedure is to take a width of the interval $d s$ that is not the same for all $s$, but that is proportional to the scale, as $[s, s+d s)=\left[s_{o}, b s_{o}\right)$, $\left[b s_{o}, b^{2} s_{o}\right), \ldots,\left[b^{k} s_{o}, b^{k+1} s_{o}\right)$, that is, $d s=(b-1) s$ (with $b>1$ ). Given a value of $s$, the corresponding value of $k$ that associates $s$ with its bin is given by $k=\left\lfloor\log _{b}\left(s / s_{o}\right)\right\rfloor$. Correspondingly, the optimum choice to assign a point to the interval $[s, s+d s]$ is given by the value $\sqrt{b} s$. This procedure is referred to as logarithmic binning, because the intervals appear with fixed width in logarithmic scale (Hergarten, 2002). In this paper we have generally taken $b \simeq 1.58$, in such a way that $b^{5}=10$, providing 5 bins per order of magnitude.
As the distributions are estimated from a finite number of data, they display statistical fluctuations. The uncertainty characterizing these fluctuations is simply related to the density by

$\frac{\sigma_{P_{S}}(s)}{P_{S}(s)} \simeq \frac{1}{\sqrt{n(s)}}$,

where $\sigma_{P_{S}}(s)$ is the standard deviation of $P_{S}(s)$ (do not confound with the standard deviation of $s$ ). This is so because $n(s)$ can be considered a binomial variable (von Mises, 1964), and then, the ratio between its standard deviation and mean fulfills $\sigma_{n}(s) /\langle n(s)\rangle \simeq 1 / \sqrt{n(s)}$, with $n(s) / N_{s} \ll 1$. As $P_{S}(s)$ is proportional to $n(s)$, the same relation holds for its relative uncertainty. 